\title{
Wine-on-tap: A study on the conservation of wines in keg Ecofass ${ }^{\circledR}$
}

\author{
B. Bach ${ }^{1}$, P. Rébénaque ${ }^{1}$, M. Sarrazin ${ }^{2}$, R. Alain ${ }^{3}$, P. Sophie ${ }^{4}$, and S. Pougnet ${ }^{5}$ \\ ${ }^{1}$ Changins, Viticulture and Enology, University of Applied Sciences, Western Switzerland, route de duillier 50, 1260 Nyon, Switzerland \\ ${ }^{2}$ BIBARIUM, Rue Dizerens 5, 1205 Genève, Switzerland \\ ${ }^{3}$ CG INDUSTRY, 17 Rue Charles Favre, 39260 Moirans-en-Montagne, France \\ ${ }^{4}$ Institut Français de la Vigne et du Vin - IFV \& SICAREX Beaujolais, 905 Route du Château de l'Eclair, 69400 Liergues, France \\ ${ }^{5}$ Ecole Hôtelière de Lausanne, HES-SO, University of Applied Sciences Western Switzerland
}

\begin{abstract}
As more and more consumers around the world integrate the choice of healthier, more ethical and eco-friendlier food and beverages in their lifestyle, the wine industry is pushed to develop more sustainable production, distribution and consumption solutions. In search of wine preservation improvements that would have both economic and ecological positive impacts, the wine industry has been investing resources in research, development and innovation regarding alternative packaging and distribution solutions for years. The keg Ecofass ${ }^{\circledR}$ is made of recyclable plastic material, high density polyethylene (HDPE) in which there is an aluminium pouch containing the liquid food. It was a question of knowing if this type of container was adapted to the conservation of the wines during several months. The method of storage and distribution has also been studied to know the impact on the oenological and sensory quality of the wines. Likewise, the nature of the pressure gas (nitrogen or compressed air) has also been tested. The contents of free and total $\mathrm{SO}_{2}$ and $\mathrm{CO}_{2}$ were monitored, and sensory analysis tests were carried out. Nevertheless, at the sensory level, the judges were able to note significant differences between the wines.
\end{abstract}

\section{Introduction}

Le vin n'est pas une boisson comme les autres : elle est au cœur de tout un écosystème dans les régions viti-vinicoles. C'est, dans ce cadre, que de plus en plus de consommateurs du monde entier intègrent le choix d'aliments et de boissons plus sains, plus éthiques et plus respectueux de l'environnement dans leur style de vie. L'industrie vinicole est amenée à développer des solutions de production, de distribution et de consommation plus durables. Bien que l'industrie du vin soit indéniablement engagée dans la voie de la durabilité, de plus en plus de travaux universitaires montrent également un intérêt croissant pour les aspects écologiques, en particulier en tant qu'avantage concurrentiel pour les parties prenantes de l'industrie du vin. À la recherche d'améliorations de la conservation du vin qui auraient des retombées positives à la fois économiques et écologiques, l'industrie vinicole investit depuis des années dans la recherche, le développement et l'innovation pour trouver des solutions alternatives de conditionnement et de distribution.

Le Bag-In-Box ${ }^{\circledR}$ (BIB) a été une première réponse. Actuellement, il est largement plébiscité par les cafés, hôtels et restaurants (CHR) ainsi que par les professionnels de la filière vinicole. Ce contenant a réalisé une véritable explosion sur le marché des vins depuis une vingtaine d'années. En effet, il présente de nombreux avantages, notamment offrant le service du vin au verre tout en assurant une conservation des vins sur plusieurs semaines après ouverture grâce à sa poche rétractable. Ce système permet une vidange sans entrée d'air, limitant ainsi les phénomènes d'oxydation. Cependant, le système BagIn-Box ${ }^{\circledast}$ connait aussi ses limites. Une des premières limites est liée à la poche souple qui ne permet pas d'utiliser des vins à des teneurs en $\mathrm{CO}_{2}$ supérieures à $1 \mathrm{~g} / \mathrm{L}$. De plus, pour le service des vins blancs et des vins rosés, les BIB doivent être placés soit en chambre froide, soit au réfrigérateur, afin que le vin soit servi à la bonne température. Cela demande, auprès des cafés, hôtels et restaurants, des investissements et des contraintes logistiques qui peuvent freiner l'utilisation de cette solution [1].

Une autre piste, qui a été explorée, a été l'utilisation de fûts inox normalement dédiés à la distribution de la bière. La possibilité de servir le vin à la tireuse comme la bière s'est révélé rapidement intéressante mais cette solution ne pouvait convenir qu'au grosse structure qui pouvait bien gérer en terme logistique, notamment sur les aspects de nettoyage. De plus, une fois percés, les fûts de ce type ont une durée de vie très limitée, le gaz de tirage $\left(\mathrm{CO}_{2}\right.$ alimentaire) étant en contact direct avec le liquide.

La solution est venue du monde de la microbrasserie qui ne pouvait se permettre de gérer un parc de fût inox et pour laquelle ont été développées des solutions hybrides entre le fût inox et le BIB. Ainsi, plusieurs sociétés ont développé des fûts en plastique munis de poches internes contenant le liquide. Dans ces systèmes, le gaz de tirage n'est plus en contact avec le liquide. Il exerce une pression entre la coque rigide et la poche, entrainant la sortie du liquide. Le fait d'utiliser un système de poche jetable évite l'étape délicate du nettoyage, l'utilisateur repartant avec une poche neuve. 


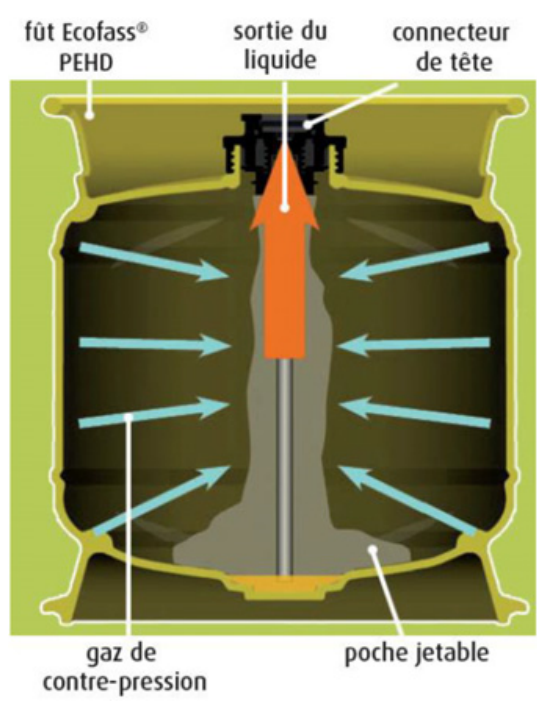

Figure 1. Présentation d'un fût Ecofass ${ }^{\circledR}$.

(source: www.ecofass.com)

Tableau 1. Composition des vins des essais.

\begin{tabular}{|c|c|c|}
\hline Analyse WineScan & Chasselas 2017 & Gamay 2017 \\
\hline Ethanol (\% vol) & 12 & 12.5 \\
\hline $\mathrm{pH}$ & 3.4 & 3.5 \\
\hline Acidité totale (T. g/L) & 3.83 & 5.34 \\
\hline Acide acétique (g/L) & 0.58 & 0.62 \\
\hline Acide tartrique (g/L) & 1.74 & 2.2 \\
\hline Acide malique (g/L) & $<0.3$ & $<0.3$ \\
\hline Acide lactique (g/L) & 1.56 & 2.00 \\
\hline Glucose-Fructose (g/L) & $<1$ & $<1$ \\
\hline Glycérol (g/L) & 5.1 & 7.2 \\
\hline Extrait sec (g/L) & 18.54 & 28.26 \\
\hline
\end{tabular}

Le fût Ecofass $^{\circledR}$ est fabriqué en matière plastique recyclable, polyéthylène haute densité (PEHD) dans lequel se trouve une poche en aluminium contenant le liquide alimentaire. En 2017, un consortium a décidé de mener une étude sur ce type de contenant dans le cadre du projet Interreg V : le projet ECOFASS-vin. Il s'agissait de savoir si ce type de contenant était adapté à la conservation et à la consommation des vins durant plusieurs mois au niveau de la qualité œnologique et sensorielle. Le mode de stockage et de tirage a également été étudié. ainsi que la nature du gaz de contre-pression $\left(\mathrm{N}_{2}\right.$ ou air comprimé). Les teneurs en $\mathrm{SO}_{2}$ libre/total et $\mathrm{CO}_{2}$ ont été suivies, et des tests d'analyses sensorielles ont été réalisés finalement au bout de 6 mois.

\section{Matériel et méthodes}

\subsection{Vins}

Le choix des vins de l'étude s'est porté sur un vin rouge de type gamay, cépage connu comme étant sensible à l'oxydation et un vin blanc de type chasselas comptant une teneur en $\mathrm{CO}_{2}$ de $1.2 \mathrm{~g} / \mathrm{L}$ (Tableau 1).

Le choix des vins s'est fait sur la base d'analyses œnologiques ( $\mathrm{SO}_{2}$ libre $20 \mathrm{mg} / \mathrm{L}, \mathrm{SO}_{2}$ total $80 \mathrm{mg} / \mathrm{L}$ ).

\subsection{Matériels}

Le volume de contenance des fûts étudiés dans le cadre de cet essai était de $10 \mathrm{~L}$. Pour le besoin de l'étude, deux types de poches ont été testées. Les poches de type PETMET qui utilisent un film barrière constituées de 4 films dont un à base de polyester métallisé :

- Film extérieur : polyester permettant la résistance aux contraintes mécaniques (PET)

- Film intermédiaire : polyester recouvert d'une couche d'aluminium (ALU).

- Film intermédiaire : polyamide (PA) obtenu par polymérisation d'un acide aminé et d'un lactame (caractérisé par une fonction amide).

- Film intérieur : polyéthylène linéaire basse densité (PELBD), utilisé en contact de l'aliment puisque chimiquement inerte.

Cette formulation est souvent destinée aux produits avec contre-pression [2], ce type de film étant connu comme étant assez étanche au gaz (entrée $\mathrm{O}_{2}$, perte $\mathrm{CO}_{2}$ ).

Les poches de type EVOH utilisent un film barrière EVOH constitué d'un triplex à base de polyester non métallisé :

- Film extérieur : polyéthylène linéaire basse densité (PELBD).

- Film intermédiaire : polyéthylène et barrière en éthyle, vinyle, alcool coextrudés (EVOH).

- Film intérieur : polyéthylène linéaire basse densité (PELBD) [3].

\subsection{Méthodologies}

Afin d'étudier l'impact œnologique et sensoriel de la conservation et de la consommation des vins en fût, l'essai s'est déroulé sur 6 mois. Pour cette étude, les deux types de poches ont été testées : poche PETMET (modalité $\mathrm{AL}$ ) et poche EVOH (modalité EV). Deux conditions de température de stockage ont été également pratiquées : une température de bouteiller de $15^{\circ} \mathrm{C}$ (modalité BT) et une température de conservation élevée de $30^{\circ} \mathrm{C}$ (modalité HT). Dans chaque scénario, deux modalités de gaz en contre-pression (1 bar) sont également mises en place en utilisant soit de l'air (modalité OX), soit de l'azote (modalité AZ). Pour chaque modalité, des triplicatas ont été réalisés.

Durant les premiers mois, des suivis analytiques ont été réalisés (Analyse WineScan, $\mathrm{SO}_{2}$ et $\mathrm{CO}_{2}$ ).

\subsection{Analyses}

\subsubsection{Analyses chimiques}

Le WineScan FOSS est un analyseur multi-paramètres de la composition chimique du vin. Les mesures sont réalisées à l'aide de la spectroscopie infrarouge par transformée de Fourier (IRTF) permettant d'analyser un échantillon liquide. Il nous permettra d'analyser des paramètres tels que le $\mathrm{pH}$, le pourcentage d'éthanol, [Acide Malique], [Acide Tartrique], [Acidité volatile], [Acidité Totale], [Glucose + Fructose]. Les teneurs en free $\mathrm{SO}_{2}$ et en $\mathrm{CO}_{2}$ ont été analysées suivant les méthodes officielles de l'OIV [3]. 


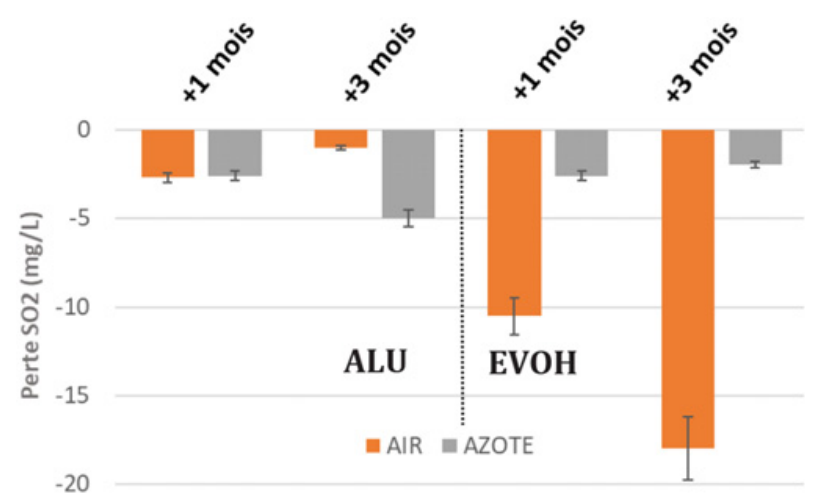

Figure 2. Somme des pertes en $\mathrm{SO}_{2}$ libre des deux vins pendant une conservation à $30^{\circ} \mathrm{C}$ pendant une durée de 1 et 3 mois.

\subsection{Analyses sensorielles}

Les analyses sensorielles sont réalisées en verre INAO dans une salle de dégustation respectant la norme ISO 8589 par le panel expert de l'école de Changins (Suisse). Les résultats sont obtenus à chaque séance de panel par le logiciel FIZZ (Biosystèmes, Couternon, France). Ils sont ensuite traités grâce au logiciel Minitab qui nous permet de savoir via l'analyse des variances ANOVA à 2 facteurs (variables explicatives juge et produit) si un descripteur est significatif ou non ( $p$-value $<0.10)$. En cas de descripteur significatif, une comparaison multiple de moyenne avec la méthode de Tukey est réalisée. Par la suite, des Analyses en Composantes Principales (ACP) sont établies par le biais du logiciel R (R Development Core Team, 2005) lorsque plusieurs descripteurs significatifs nous permettent de déterminer une différence ou une similarité au niveau des produits.

\section{Résultats et discussion}

\subsection{Analyses chimiques durant la conservation}

$\mathrm{Au}$ bout de 1 et 3 mois, des échantillons ont été prélevés dans les fûts conservés à $30^{\circ} \mathrm{C}$ considérant qu'il s'agissait de la condition la plus oxydante et donc la plus consommatrice de $\mathrm{SO}_{2}$ (Fig. 2).

La perte en $\mathrm{SO}_{2}$ libre sur les vins protégés par la poche de type PETMET (ALU) a été limitée avec un très faible impact du gaz de tirage. Au contraire, les vins protégés par la poche de type EVOH ont vu leur teneur en $\mathrm{SO}_{2}$ libre largement impactée. Ce résultat est cohérent car il est connu que la perméabilité des films EVOH est plus importante que les films de type PETMET [4]. Le fait d'utiliser de l'azote comme gaz de tirage a un effet protecteur important car on retrouve les valeurs obtenues avec les poches PETMET.

Les teneurs en $\mathrm{CO}_{2}$ ont également pu être mesuré sur le vin blanc (Fig. 3).

En ce qui concerne les vins conservés à $15^{\circ} \mathrm{C}$, aucune variation significative n'a pu être observée. Au contraire, la conservation à $30^{\circ} \mathrm{C}$ a affecté les teneurs en $\mathrm{CO}_{2}$ des vins indépendamment du type de poche et du type de gaz de tirage.

Il est à remarquer que les autres paramètres ont pu être testés (analyses winescan). Aucune différence n'a pu être observé entre les modalités.

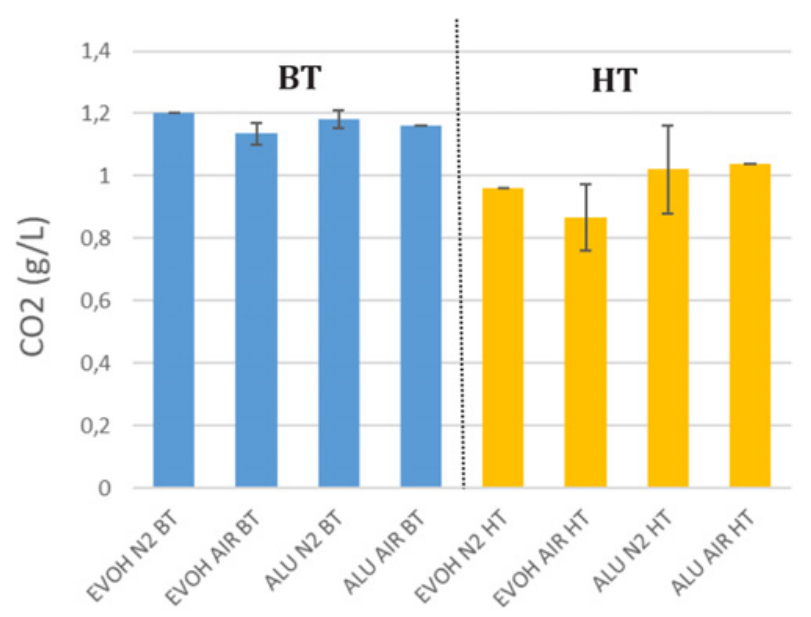

Figure 3. Suivi du $\mathrm{CO}_{2}$ du vin blanc (chasselas) sur une durée de 3 mois à une température de conservation de $15^{\circ} \mathrm{C}(\mathrm{BT})$ et $30^{\circ} \mathrm{C}(\mathrm{HT})$.
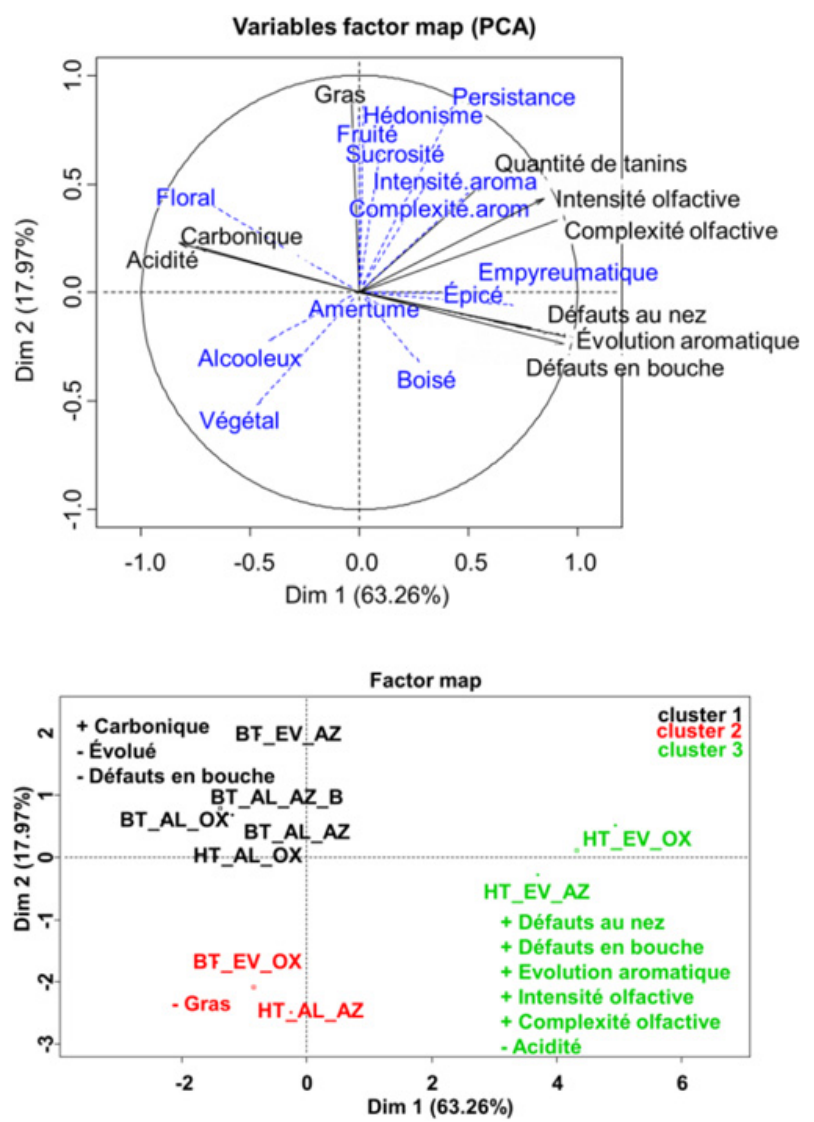

Figure 4. Cercle de corrélation et carte des individus issus de l'ACP obtenu lors de l'analyse sensorielle du vin blanc de type chasselas.

\subsection{Analyses sensorielles au bout de 6 mois}

Les analyses sensorielles des différentes modalités ont montré des différences significatives. En ce qui concerne le vin blanc de type chasselas, les différences les plus marquées ont été trouvées sur les aspects «carbonique » du vin (Fig. 4).

Ce résultat peut être mis en relation avec les pertes de $\mathrm{CO}_{2}$ mesurées sur les modalités conservées à $30^{\circ} \mathrm{C}$. Il est à remarquer que les vins issus des poches de type PETMET (ALU) ont conservé cet aspect, se retrouvant 

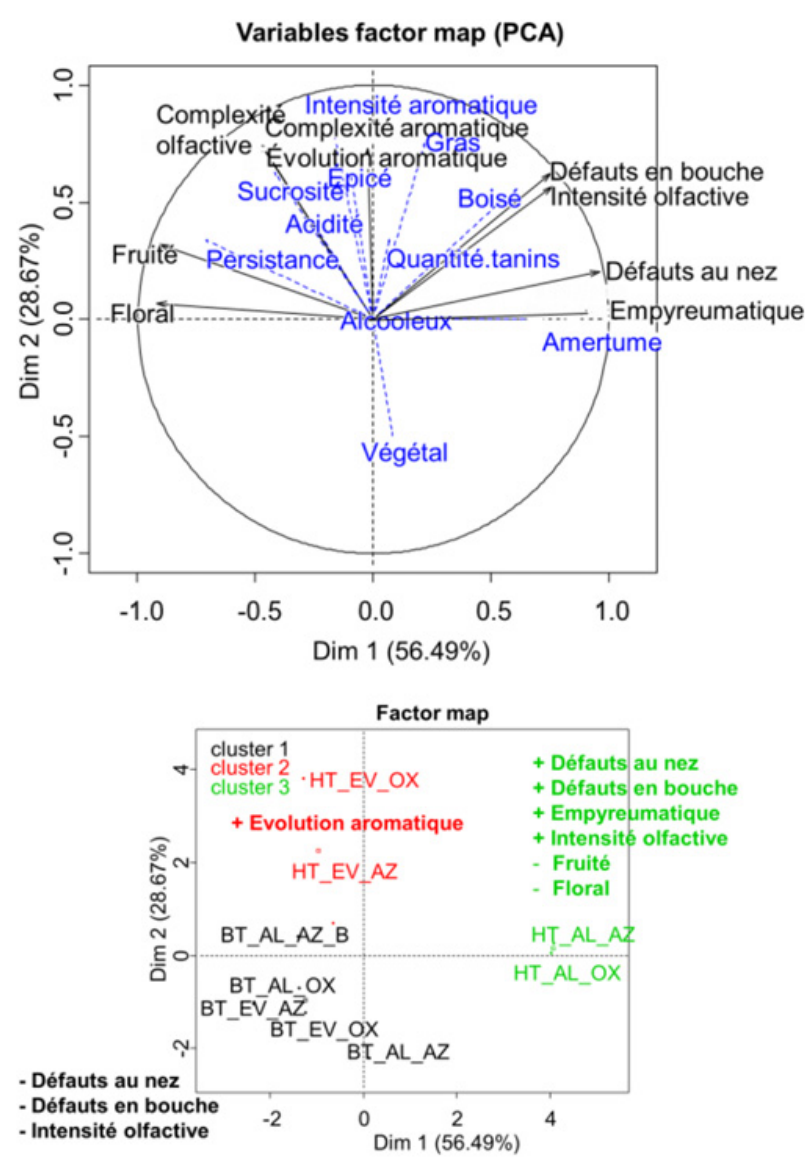

Figure 5. Cercle de corrélation et carte des individus issus de l'ACP obtenu lors de l'analyse sensorielle du vin rouge de type gamay.

dans la même zone sensorielle que les vins conservés à $15^{\circ} \mathrm{C}$. Au contraire, les vins conservés dans les poches de type $\mathrm{EVOH}$ ont vu leur profil organoleptique très affecté. Néanmoins, à température de $15^{\circ} \mathrm{C}$, le fait d'utiliser de l'azote comme gaz de tirage a permis de limiter la perméabilité du film EVOH. A $30^{\circ} \mathrm{C}$, l'effet protecteur de l'azote n'est cependant plus efficace.

En ce qui concerne l'analyse des vins rouges de type gamay, les résultats de l'analyse sensorielle sont plus contrastés (Fig. 5).

En effet, dans le cas de ce vin, la température de conservation de $30^{\circ} \mathrm{C}$ semble avoir affecté négativement le profil organoleptique du vin, ceci indépendamment du type de poche et du type de gaz de tirage. Contrairement à ce qui pu être établi avec le vin blanc, il semblerait que dans le cas du vin rouge les poches de type PETMET
(ALU) aient donné les vins avec le moins bon potentiel de dégustation (présence de défauts, moins fruité, moins floral). Au contraire, les vins issus des poches de type EVOH se sont trouvés être plus évolués mais sans que cela soit traduit comme un défaut. Il semblerait donc que dans ce cas, l'oxygénation amenée par le film de type EVOH s'est révélée bénéfique au vu des températures appliqués. Pour une conservation à $15^{\circ} \mathrm{C}$, peu de différences ont pu être établies entre les différentes modalités de type de poche et de type de gaz de tirage.

En conclusion, les résultats obtenus au cours de cette expérimentation sont très positifs. Néanmoins, au niveau sensoriel, les juges ont pu noter des différences significatives entre les vins. En effet, suivant les scénarios, les caractéristiques des vins ont évolué sur la durée de l'essai. Le type de gaz (air ou azote) ainsi que le type de film utilisé a un impact sur la qualité du vin lors de son stockage ou sa conservation. Nous avons pu démontrer que le film de type EVOH pouvait avoir les mêmes capacités de protection que le film de type PETMET si l'azote était employé comme gaz de tirage. Cet élément se révèle être un point important quand on connait le bénéfice en terme écologique et économique que peut avoir l'utilisation de film de type EVOH.

Les tests à température élevée $\left(30^{\circ} \mathrm{C}\right)$ sur une période longue (6 mois) ont montré la bonne protection du matériel. Dans ce cadre, la poche de type PETMET ont mieux protéger le vin, notamment sur les aspects de préservation de gaz (entrée $\mathrm{O}_{2}$, perte $\mathrm{CO}_{2}$ ). Ces résultats sont encourageants car ils pourraient permettre d'envisager de réduire les doses de $\mathrm{SO}_{2}$.

Ce projet bénéficie d'un financement du programme Interreg $\mathrm{V}$ France-Suisse qui est un programme de coopération territoriale européenne, mis en œuvre sur un territoire de coopération réparti entre la France et la Suisse.

\section{Références}

[1] A. Peychès-Bach, Interactions Vin/Emballage/ Environnement Application au Bag-In-Box ${ }^{\circledR}$. Thèse de doctorat (2009)

[2] P. Chomon, Les complexes souples ou rigides utilisés en emballage. Techniques de l'Ingénieur AM3577. (2006)

[3] C.M. Plank, B.C. Trela, Am. J. Enol. Vitic. 69, 307 (2018)

[4] OIV, "Recueil des méthodes internationales d'analyse des vins et des moûts" Paris (2011)

[5] Y. Fu, L.-T. Lim, P.D. McNicholas, J. Food Sci. 74, C608 (2009) 